\title{
La goutte
}

Timothy S.H. Kwok MD, Victoria Y.Y. Xu MD, Shirley L. Lake MD MSc

Citation : CMAJ 2021 February 1;193:E171. doi : 10.1503/cmaj.201392-f

Voir la version anglaise de l'article ici : www.cmaj.ca/lookup/doi/10.1503/cmaj.201392

\section{La prévalence de la goutte est en hausse, et de nombreux patients présentent des comorbidités courantes}

La prévalence de la goutte est d'environ $4 \%$, ce qui correspond à une augmentation de $59 \%$ au cours de la dernière décennie ${ }^{1}$. Plus de $60 \%$ des patients atteints de la goutte présentent aussi un syndrome métabolique, ce qui devrait être pris en compte lors de l'évaluation. Les facteurs de risque de la goutte sont les suivants : sexe masculin, âge avancé, insuffisance rénale chronique, déshydratation (incluant traitement diurétique), hyperuricémie due à un renouvellement cellulaire rapide, (p. ex., hémolyse, chimiothérapie) et consommation excessive de purines (annexes $1 \mathrm{~A}$ et $\mathrm{B}$, accessibles en anglais au www. cmaj.ca/lookup/doi/10.1503/cmaj.201392/tab-related-content)2 .

2 Les crises de goutte sont généralement monoarticulaires et atteignent leur intensité maximale en quelques heures Les crises surviennent souvent la nuit et affectent les articulations distales (p. ex., première articulation métatarsophalangienne). Les antécédents de traumatisme articulaire et les anomalies dégénératives en sont des facteurs prédisposants (annexe 1C). La présence d'érythème et de chaleur périarticulaires et d'une desquamation localisée peuvent faire penser à une infection ${ }^{2}$. Le diagnostic différentiel de la crise de goutte aiguë inclut l'arthrite septique, l'arthrite post-traumatique et l'hémarthrose, surtout chez les patients sous anticoagulothérapie. Contrairement à la goutte, l'arthrose tend à être une maladie chronique, et l'arthrite inflammatoire est généralement polyarticulaire².

\section{Le diagnostic repose sur la détection de cristaux durant \\ l'analyse du liquide synovial}

L'examen au microscope révèle la présence de cristaux d'urate monosodique à biréfringence négative. La goutte et l'arthrite septique se manifestent parfois concomitamment; il est donc important d'écarter toute possibilité d'infection par mise en culture et coloration de Gram. La radiographie peut révéler la présence de tophi ou d'érosions à bords surplombants. Le taux sérique d'acide urique est parfois élevé, mais un taux normal ne permet pas d'écarter le diagnostic de goutte. Une règle diagnostique validée peut faciliter le diagnostic sans arthrocentèse (annexe $1 \mathrm{~A})^{3}$.

\section{Un traitement pour la goutte administré dans les 24 heures suivant l'apparition des symptômes permet de réduire l'intensité et la durée de la crise \\ L'administration immédiate de la pharmaco- thérapie dépend des comorbidités du patient. Les anti-inflammatoires non stéroïdiens, la colchicine et les corticostéroïdes sont les traitements de première intention (annexe 1D). La prise d'hypo-uricémiants ne devrait pas être interrompue durant les crises $^{4}$.}

Il faut commencer un traitement hypo-uricémiant chez les patients atteints de goutte tophacée s'ils présentent des signes de goutte à la radiographie ou s'ils ont $\geq \mathbf{2}$ crises de goutte par année

Il faut ajuster la dose d'hypo-uricémiant (annexe 1D) pour atteindre un taux d'urate sérique $<360 \mu \mathrm{mol} / \mathrm{L}$ en effectuant des contrôles tous les mois. Des taux d'urate sérique $<360 \mu \mathrm{mol} / \mathrm{L}$ favorisent la dissolution des cristaux et réduisent d'environ $80 \%$ le risque de nouvelles crises $^{5}$. Un traitement prophylactique contre la goutte à court terme à la colchicine, au naproxène ou à la prednisone devrait être prescrit pour les 3-6 premiers mois de traitement hypo-uricémiant (annexe 1D). Perdre du poids (c.-à-d. réduire l'indice de masse corporelle de $>5 \%$ ), limiter la consommation d'alcool $(<1-2$ unités/j) et réduire l'apport en fructose $(<1 \mathrm{~g} / \mathrm{kg} / \mathrm{j})$ et en purines peut aider à abaisser le taux d'urate ${ }^{4}$. Selon le cas, on envisagera des approches multidisciplinaires et des consultations auprès de spécialistes. 


\section{Références}

1. Rai SK, Aviña-Zubieta JA, McCormick N, et al. The rising prevalence and incidence of gout in British Columbia, Canada: population-based trends from 2000-2012. Semin Arthritis Rheum 2017;46:451-6.

2. Dalbeth N, Merriman TR, Stamp LK. Gout. Lancet 2016;388:2039-52.

3. Janssens HJ, Fransen J, van de Lisdonk EH, et al. A diagnostic rule for acute gouty arthritis in primary care without joint fluid analysis. Arch Intern Med 2010;170:1120-6.

4. FitzGerald JD, Dalbeth N, Mikuls T, et al. 2020 American College of Rheumatology guideline for the management of gout. Arthritis Care Res (Hoboken) 2020;72:744-60.

5. Shoji A, Yamanaka $H$, Kamatani N. A retrospective study of the relationship between serum urate level and recurrent attacks of gouty arthritis: evidence for reduction of recurrent gouty arthritis with antihyperuricemic therapy. Arthritis Rheum 2004;51:321-5.

\section{Intérêts concurrents : Aucun déclaré}

Cet article a été révisé par des pairs.

Affiliations : Département de médecine (Kwok, Xu, Lake) et divisions de médecine gériatrique (Xu) et de rhumatologie (Lake), Université de Toronto; Division de rhumatologie (Lake), Centre des sciences de la santé Sunnybrook, Toronto, Ont.

Remerciements : Les auteurs souhaitent remercier les $D^{\text {rs }}$ Lucille Chan, Betty Lee et Mark Leung pour leurs commentaires constructifs sur les premières versions de ce manuscrit.

Propriété intellectuelle du contenu : Il s'agit d'un article en libre accès distribué conformément aux modalités de la licence Creative Commons Attribution (CC BY-NC-ND 4.0), qui permet l'utilisation, la diffusion et la reproduction dans tout médium à la condition que la publication originale soit adéquatement citée, que l'utilisation se fasse à des fins non commerciales (c.-à-d., recherche ou éducation) et qu'aucune modification ni adaptation n'y soit apportée. Voir : https://creativecommons.org/licenses/by-nc-nd/4.0/deed.fr.

Correspondance : Shirley Lake, shirley.lake@sunnybrook.ca 